\title{
HASTES INSTRUMENTADAS PARA A MENSURAÇÃO DA RESISTÊNCIA MECÂNICA DO SOLO
}

\author{
VIACHESLAV I. ADAMCHUK ${ }^{1}$, JOSÉ P. MOLIN ${ }^{2}$
}

RESUMO: A presença de camada de solo compactada pode significar sérios problemas ao desenvolvimento do sistema radicular das culturas em geral. A correção pode ser feita por métodos biológicos ou mecânicos. Uma das formas de diagnóstico da sua presença é com o uso de penetrômetros que exigem tempo considerável para a obtenção de dados. Com o advento dos recursos de espacialização das informações na forma de mapas, a necessidade de amostragem passou a ser ainda maior. Este trabalho reporta a busca de uma solução alternativa ao uso do penetrômetro. Envolve a geração de mapas de resistência mecânica do solo ao deslocamento de uma haste em três profundidades, baseado em três hastes instrumentadas, um receptor de GPS e um sistema de aquisição de dados. O sistema é acoplado a uma barra porta-ferramenta montada no engate de três pontos do trator. A configuração mostrou ser mecanicamente bastante simples e confiável. Dados de um teste inicial em campo demonstraram que o sistema pode produzir mapas que denotam a variabilidade espacial da referida resistência nas áreas amostradas, com alta resolução, densidade de amostragem elevada e com capacidade operacional ainda mais elevada se comparada com levantamentos feitos com métodos convencionais.

PALAVRAS CHAVE: agricultura de precisão, sensores de solo, compactação.

\section{INSTRUMENTED SHANKS FOR SOIL MECHANICAL RESISTANCE MEASUREMENTS}

ABSTRACT: The presence of soil compaction may be considered a serious problem for the development of crop root systems. It can be alleviated by biological or mechanical methods (such as tillage). One of the most popular diagnostic methods is based on standardized cone penetrometer measurements, which requires a considerable amount of time to obtain the data. With the advent of site-specific field management, high-density measurements have become a necessity. This work reports on an alternative approach to use a cone penetromenter. It involves the generation of maps of soil mechanical resistance at three depths using three instrumented blades, a GPS receiver and a data logger. The system is attached to a toolbar that is installed to the tractor's three-point hitch. It has proven to be mechanically simple and reliable. Maps indicating the variability of soil mechanical resistance with relatively high spatial resolution (as compared to traditional methods) were generated in a reasonable amount of time based on data collected during initial field tests.

KEYWORDS: precision agriculture, soil sensor, compaction.

\section{INTRODUÇÃO}

Tradicionalmente, um mesmo tratamento ou cultivo tem sido aplicado a uma lavoura inteira. $\mathrm{O}$ gerenciamento e o tratamento localizado, ao contrário, propõem a aplicação não-uniforme de insumos e de práticas de preparo do solo para contemplar a variação de condições desse no campo. O cultivo a profundidades variáveis fornece a potencialidade de controlar a compactação de solo e a conservar energia requerida para o cultivo (MORGAN \& ESS, 1997). GORUCU et al. (2001) obtiveram até

\footnotetext{
${ }^{1}$ Eng ${ }^{0}$ Agrícola, Professor, Departamento de Engenharia de Sistemas Biológicos, Universidade de Nebraska-Lincoln, 212 LW Chase Hall, Lincoln, NE 68583-0726, 402-472-6338, vadamchuk2@unl.edu.

${ }^{2}$ Engo ${ }^{\circ}$ Agrícola, Professor, Departamento de Engenharia Rural, ESALQ/USP, Piracicaba - SP, jpmolin@esalq.usp.br

Recebido pelo Conselho Editorial em: 10-12-2004

Aprovado pelo Conselho Editorial em: 4-4-2006
} 
$42,8 \%$ de economia de energia e $28,4 \%$ de economia de combustível quando utilizaram o recurso de cultivo a profundidades variáveis.

Em sistemas de semeadura direta tem sido observado aumento da densidade do solo superficial, tendo como conseqüência maior resistência à penetração ou índice de cone, detectada por autores como SANTOS (1993) de 0,00 a 0,05 m, KLEIN (1998) de 0,00 a 0,40 m, BERTOL et al. (2001) de 0,000 a 0,025 m, SILVA JUNIOR (2001) de 0,10 a 0,20 m.

A Sociedade Americana de Engenharia Agrícola (ASAE) desenvolveu um padrão (ASAE, 2000a) e uma prática de engenharia (ASAE, 2000b) na aplicação de um penetrômetro - padrão de cone para medir a resistência mecânica do solo sob penetração vertical. Esse método - padrão tem diversas limitações devido à variação significativa da medida e à densidade espacial economicamente praticável relativamente baixa da medida. Consequientemente, novos métodos automatizados para substituir as medidas do penetrômetro de cone têm sido desenvolvidos.

ROONEY et al. (2002) adicionaram um sensor de atrito e um sensor óptico para operar hidraulicamente um penetrômetro de cone. RAPER et al. (1999) criaram uma sonda de múltiplos penetrômetros de cone montada na parte traseira de um trator para automatizar o processo de amostragem e permitir o cálculo da média de cinco medidas simultâneas. ALIHAMSYAH et al. (1990) utilizaram um penetrômetro de cone para medir horizontalmente a resistência mecânica do solo em tempo real, que aumentou significativamente a densidade espacial da medida. Seu sistema utilizou uma haste com uma ponta cônica ligada a uma célula de carga. GLANCEY et al. (1996) e EHRHARDT et al. (2001) utilizaram a força de tração de um implemento como indicador da resistência mecânica do solo, nesse caso, mensurada com as hastes do implemento rompendo o solo no sentido horizontal. SIRJACOBS et al. (2002) usaram um anel transdutor octogonal numa barra portaferramenta montada para medir forças horizontais e verticais, assim como o momento torçor produzido pela haste quando puxada através do solo. Uma haste instrumentada similar foi desenvolvida por OWEN et al. (1987).

Em outro projeto, uma haste equipada com extensômetros de resistência elétrica ("strain gages") e um potenciômetro foram projetados para mover para cima e para baixo continuamente ao ser puxada através do solo (MANOR \& CLARK, 2001). Esse sistema foi desenvolvido para detectar a profundidade da camada de impedimento do solo. GLANCEY et al. (1989) projetaram um escarificador instrumentado para medir a resistência mecânica do solo em diferentes profundidades no campo. Seu projeto incluiu seis extensômetros de resistência elétrica montados diretamente atrás da borda cortante frontal e outros seis na parte traseira da haste, na mesma profundidade que os extensômetros frontais. Um sensor adicional foi instalado para medir as forças verticais que agem no escarificador. Similarmente, ADAMCHUK et al. (2001) usaram uma haste lisa vertical com extensômetros de resistência elétrica dispostos em diferentes níveis na parte traseira da haste. ANDRADE et al. (2001) usaram uma haste equipada com células de carga para medir a resistência mecânica do solo a uma profundidade de $0,60 \mathrm{~m}$ em incrementos de $0,075 \mathrm{~m}$ e, para tanto, utilizaram uma célula de carga separada para cada profundidade.

As principais limitações dos sensores dos sistemas acima referenciados incluem medida a uma única profundidade, distúrbio excessivo do solo, sinal baixo em relação ao ruído e custo potencial elevado. Conseqüentemente, um sistema simples e confiável para mapear a resistência mecânica do solo em várias profundidades e em tempo real é necessário.

O objetivo deste trabalho foi desenvolver um sistema com essa característica, capaz de mapear a resistência mecânica do solo, em tempo real, em três profundidades simultaneamente e testá-lo em condições locais. 


\section{MATERIAL E MÉTODOS}

Um sistema de múltiplas hastes foi projetado para permitir a medida simultânea da resistência mecânica do solo em três profundidades, ao deslocar-se no campo. As pressões de resistência mecânica do solo, uniformemente distribuídas (p1, p2 e p3), foram calculadas baseadas na carga aplicada a cada haste que age como uma viga em balanço. Um par de extensômetros de resistência elétrica (EA-06-250PD-350, Measurements Group, Inc. ${ }^{\circledR}$ ) foi instalado a 0,36 $\mathrm{m}$ da base de cada haste no lado de tração e outro no lado de compressão de cada haste, paralelos entre si e configurados em uma ponte de Wheatstone completa, permitindo a medição de $0,1 \mathrm{~m}$ de profundidade por haste. Isso foi realizado instalando as hastes de modo que o segmento dos $0,10 \mathrm{~m}$ superiores da haste 2 funcionasse no entalhe aberto pela haste 1 , e segmento dos $0,20 \mathrm{~m}$ superiores da haste 3 funcionasse nos entalhes abertos pelas hastes 1 e 2 . As bordas cortantes foram feitas mais largas do que as hastes, permitindo deixar sulcos abertos para a passagem das hastes.

As três hastes utilizadas nesse sistema foram confeccionadas em aço de 12,7 mm de espessura, com tensão de tração de $690 \mathrm{MPa}$. O comprimento máximo (na direção do deslocamento) de cada haste foi escolhido a fim de produzir uma escala aceitável das tensões sob condições de cargas normais. A deformação específica mínima foi ajustada em $100 \mu \mathrm{m} \mathrm{m}^{-1}$ para fornecer uma relação aceitável entre sinal e ruído, e a deformação específica máxima foi ajustada em $600 \mu \mathrm{m} \mathrm{m}^{-1}$ para evitar danos aos extensômetros.

A seguinte equação foi utilizada para descrever a relação entre a deformação específica e a resistência mecânica para cada haste:

$$
\varepsilon=\frac{6 \mathrm{a} \mathrm{b}_{1} \mathrm{y}}{\mathrm{E} \mathrm{b} \mathrm{h}^{2}} \mathrm{p} 10^{6}
$$

em que,

$\varepsilon$ - deformação específica medida, $\mu \mathrm{m} \mathrm{m}^{-1}$;

E - módulo de elasticidade (para Aço E = $207 \mathrm{GPa}$ );

a - altura vertical da borda cortante, $\mathrm{mm}$;

$\mathrm{b}_{1}$ - largura de corte da borda cortante, $\mathrm{mm}$;

b - largura da haste, $\mathrm{mm}$;

h - máximo comprimento da haste (na altura dos extensômetros), $\mathrm{mm}$;

y - distância vertical entre o centro de corte da borda cortante e os extensômetros, mm, e

$\mathrm{p}$ - resistência mecânica do solo agindo sobre a borda cortante, MPa.

Uma "pressão-padrão" foi utilizada para calcular o comprimento máximo (h) para cada haste. Os valores para "pressão-padrão" em três profundidades foram os mesmos que aqueles utilizados por ADAMCHUK et al. (2001): aumentando de $1 \mathrm{MPa}$ na superfície do solo para $3 \mathrm{MPa}$ na profundidade de 0,3 m abaixo da superfície. Conseqüentemente, as pressões uniformemente distribuídas p1, p2 e p3 corresponderam a $1 \mathrm{MPa}, 2 \mathrm{MPa}$ e $3 \mathrm{MPa}$, respectivamente. Após ter simplificado a eq.(1) para $\mathrm{p}-\mathrm{k}_{\mathrm{i}} \varepsilon$, uma constante $(\mathrm{k})$ para cada haste (i) pôde ser encontrada $\left(\mathrm{k}_{1}=0,00438 ; \mathrm{k}_{2}=0,00595\right.$, e $\left.\mathrm{k}_{3}=0,00778\right)$.

A borda cortante das hastes foi construída com cunhas de $45^{\circ}$, de modo que o solo se move sobre elas uniformemente. Esse ângulo foi estabelecido de acordo com pesquisas feitas por GILL (1968), que mostraram que pontas com ângulos de 40 a $45^{\circ}$ fornecem força de corte mínima ao serem lentamente puxadas através do solo. As bordas cortantes são de $19 \mathrm{~mm}$ de largura e $100 \mathrm{~mm}$ de altura e foram termicamente tratadas para resistir ao desgaste. Os $45^{\circ}$ dos chanfros foram estendidos para cima, além das bordas cortantes nas hastes, para melhorar a eliminação de resíduos de culturas e solo. 
As três hastes foram instaladas em um único suporte fixado por meio de abraçadeira a uma barra porta-ferramentas equipada com um parafuso cisalhante e um parafuso pivotante para impedir danos às hastes sob carga excessiva. Uma quarta haste foi adicionada para medir a fricção solo-metal e não foi utilizada neste estudo. Mapas de fricção poderiam ser utilizados para estudos de variabilidade dos efeitos da textura versus umidade do solo, bem como compensar os efeitos da variabilidade espacial da fricção nas hastes 2 e 3 e que podem estar contribuindo nas mensurações.

O sistema de aquisição de dados consistiu em uma placa condicionadora de sinal (SC-2043-SG, National Instruments ${ }^{\circledR}$ ), conversor análogo para digital (DAQCard-1200, National Instruments ${ }^{\circledR}$ ) e um computador portátil. As medidas feitas com os extensômetros de resistência elétrica são coletadas a uma taxa de $150 \mathrm{~Hz}$, calculando-se a média sobre $1 \mathrm{~s}$ e são registradas em arquivo tipo texto. $\mathrm{O}$ sistema inclui ainda um receptor de GPS que fornece a posição geográfica (longitude e latitude) a cada $1 \mathrm{~s}$, gravada junto com as medidas de tensão.

Para um teste de validação do sistema, esse foi acoplado a um trator MF $5300^{\circledR}$ e submetido a teste em duas áreas de produção de cana-se-açúcar com colheita manual, pertencentes à Usina Açucareira Guaíra, na região de Guaíra - SP. A área 1, pertencente à Fazenda Nova Índia, município de Barretos, foi colhida no dia 2-5-2004, no seu quarto corte, e o teste ocorreu em 11-5-2004. Já a área 2, pertencente à Fazenda Vera Cruz, município de Guaíra, foi colhida no dia 4-5-2004, e o teste foi conduzido no dia 12-5-2004, após o segundo corte. Em ambas as áreas, os percursos foram espaçados de $14 \mathrm{~m}$, equivalente a dez ruas de $1,4 \mathrm{~m}$ e com passadas em ruas sem resíduos de ponteiras e em mesmas condições de tráfego. Na área 1 , a velocidade de deslocamento variou entre 1,5 e $1,6 \mathrm{~m} \mathrm{~s}^{-1} \mathrm{e}$ na área 2, variou entre $2,2 \mathrm{e} 2,4 \mathrm{~m} \mathrm{~s}^{-1}$.

\section{RESULTADOS E DISCUSSÃO}

O sistema é apresentado na Figura 1 e mostra as três hastes de mensuração da resistência mecânica, instrumentadas com os conjuntos de extensômetros de resistência elétrica e com as suas ponteiras de bordas cortantes, a haste de fricção, ligadas a uma abraçadeira, assim como a posição de um parafuso pivotante e outro de cisalhamento. Essa configuração é mecanicamente simples, considerando-se um sistema de coleta de dados para três camadas de solo. Todo o conjunto é montado em uma barra porta-ferramentas acoplada ao engate de três pontos do trator, na qual é adicionado um rodado para flutuação e controle de profundidade e pesos suficientes para garantir a penetração das hastes na profundidade definida. Dependendo das condições de superfície do solo em que o sistema irá atuar, é também necessário adicionar à barra porta-ferramentas um conjunto para corte e/ou remoção de resíduos vegetais.

Com essa configuração, espera-se que a primeira haste represente as condições do solo superficial. No entanto, isso só é possível se o efeito do resíduo, porventura presente na superfície, for corretamente eliminado. Para tanto, é necessária a remoção completa desse resíduo ou a anulação do seu efeito com o uso de um disco de corte que, no entanto, não pode estar alinhado com as hastes para não alterar a condição original do solo. Já a haste 2 deverá mensurar as condições do solo à profundidade crítica, por exemplo, para ambientes de semeadura direta, como detectado por MOLIN \& SILVA JÚNIOR (2004).

A haste 3 deverá identificar a presença de compactação em lavouras em que não foi feita uma correção adequada do perfil do solo quando da transição entre o sistema de preparo convencional e adoção da semeadura direta. Já em sistemas de produção como na cana-de-açúcar, o tráfego intenso de máquinas e o sistema de preparo do solo geram distribuição da compactação no perfil a profundidades ainda maiores, como observado por FONSECA et al. (1988) que, ao avaliarem o efeito da compactação do solo por colhedoras e veículos de transporte, identificaram que os maiores índices de compactação ocorreram para as camadas compreendidas entre 0,2 e $0,3 \mathrm{~m}$ e as menores compactações 
ocorreram na profundidade de 0 a $0,1 \mathrm{~m}$. Nesse caso, a solução aqui apresentada pode ter limitações ou excesso de detalhamento, tendo em vista que qualquer ação corretiva mecânica na forma de subsolagem deve atuar em todo o perfil compactado, o que normalmente ocorre de 0,1 a $0,3 \mathrm{~m}$ ou mais. Portanto, o diagnóstico de camadas distintas provavelmente não seja prioritário e a profundidade atingida não seja suficiente. Por outro lado, em culturas perenes, como o café e citros, os aspectos relativos à compactação também são de grande importância. MAGALHÃES et al. (2004) identificaram a presença de camada compactada em lavoura de café entre 0,1 e 0,2 $\mathrm{m}$ de profundidade, mensurada com penetrômetro hidráulico-eletrônico e indicaram problemas no uso de penetrômetro na presença de raízes. A utilização de hastes instrumentadas para esse fim é ainda mais agravada, pois as raízes, além de serem rompidas, podem danificar o instrumento e geram valores ireais de resistência mecânica.

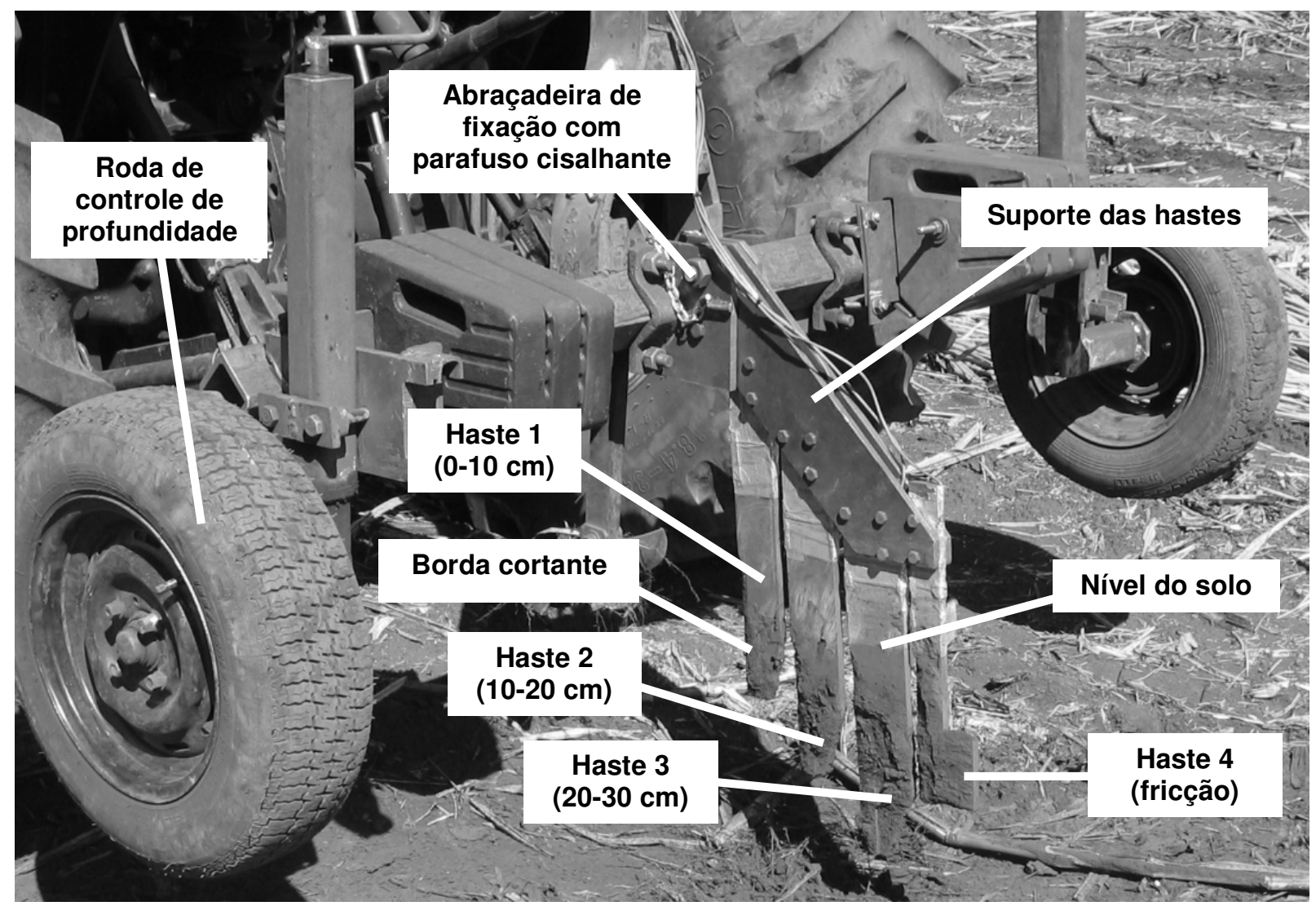

FIGURA 1. Sistema de múltiplas hastes para o mapeamento de resistência do solo na condição de trabalho nos ensaios de campo.

Em uma etapa anterior do desenvolvimento dessa solução (SIEFKEN et al., 2005), as hastes foram testadas individualmente em laboratório, montando-as em uma posição horizontal e aplicando pesos conhecidos no centro das bordas cortantes, e a tensão medida foi comparada à tensão calculada com correlação que atingiu $\mathrm{R}^{2}=0,999$, inclinação muito próxima a 1,0 e interceptação em zero (linha de 1:1). Isso confirmou, utilizando especificações da unidade condicionadora de sinal e dos extensômetros, o fator de conversão calculado de tensão para deformação específica de $52,125 \mu \mathrm{V}$ por $1 \mu \mathrm{m} \mathrm{m}^{-1}$. Na mesma etapa, em testes de campo, o sistema mostrou que a profundidade da medida afetou significativamente a relação entre as medidas mecânicas correspondentes da resistência do solo obtidas, utilizando as hastes instrumentadas e um penetrômetro de cone. A comparação das medidas obtidas utilizando as hastes instrumentadas e corrigidas para a profundidade e o penetrômetro de cone, expostos as condições dinâmicas distintas, especialmente de velocidade, mostrou correlação aceitável 
com $\mathrm{R}^{2}=0,76$. As medidas obtidas em pequenas parcelas com diferentes históricos e preparos de solo apresentaram variância dentro das parcelas significativamente mais baixa se comparada ao penetrômetro de cone. Mudanças na velocidade de deslocamento, de 0,45 a 2,24 $\mathrm{m} \mathrm{s}^{-1}$, não tiveram efeito significativo nas medidas da resistência mecânica do solo.

Na Figura 2, apresentam-se os resultados dos pontos de leitura georreferenciados, na forma de mapas com legenda única para permitir a comparação visual. Nos testes feitos nas áreas de produção de cana-de-açúcar, observa-se a identificação de grandezas de resistência mecânica com elevada variabilidade espacial, reforçada pelos indicadores da Tabela 1 que sintetiza a estatística descritiva dos dados obtidos nos testes de campo. As áreas de resistência mecânica elevada e baixa podem ser vistas em cada uma das camadas dos mapas, mas ficaram mais evidentes na camada de 0,2 a 0,3 m. Observase, por exemplo, que, na área 1, os níveis de resistência mecânica são maiores na camada de 0,0 a 0,1 m se comparado com os valores observados na área 2, embora ambos sejam inexpressivos sob o ponto de vista de diagnóstico da presença de compactação. A camada compreendida entre 0,1 e $0,2 \mathrm{~m}$, em ambas as áreas, apresentou valores intermediários, e a camada mais profunda $(0,2$ a $0,3 \mathrm{~m})$, mensurada pela haste 3, resultou nos valores mais elevados de resistência mecânica, indicando os valores mais elevados de compactação para essa camada do solo.

Os mapas ilustram que o sistema de hastes instrumentadas pode produzir mapas de alta resolução em áreas de produção. A densidade de amostragem nesse caso, com espaçamento de $14 \mathrm{~m}$ e com velocidade de deslocamento variando de 1,5 a 2,4 $\mathrm{m} \mathrm{s}^{-1}$, foi de 48 e 61 pontos por ha nas áreas 1 e 2, respectivamente. Além disso, os pontos representam a integração de média de $1 \mathrm{~s}$ de coleta, o que, por si, já é mais representativo que a amostragem com penetrômetros. Esses, por sua vez, têm sido utilizados com densidades amostrais, por exemplo, de 16 pontos por ha (MOLIN \& SILVA JÚNIOR, 2004), 30 pontos por ha (LANÇAS et al., 2000) e 32 pontos por ha (MAGALHÃES et al., 2004) em trabalhos experimentais e com produtividade baixa para padrões de serviço em escala comercial. Para a condição dos testes de campo, a capacidade operacional em valores aproximados variou de 6 a $9 \mathrm{ha}^{-1}$. Obviamente, todos esses indicadores são fortemente afetados pelos parâmetros espaçamento e velocidade.

TABELA 1. Estatística descritiva para os valores de resistência mecânica (MPa) medidos com as três hastes instrumentadas nas duas áreas experimentais.

\begin{tabular}{|c|c|c|c|c|c|}
\hline Profundidade da Camada $(\mathrm{cm})$ & Valor Mínimo & Valor Máximo & Média & Desvio-Padrão & C.V. (\%) \\
\hline \multicolumn{6}{|c|}{ Área 1} \\
\hline 0 a 10 & 0,00 & 0,78 & 0,20 & 0,10 & 51,10 \\
\hline 10 a 20 & 0,20 & 1,77 & 0,79 & 0,27 & 34,06 \\
\hline 20 a 30 & 0,33 & 2,64 & 1,12 & 0,38 & 33,42 \\
\hline \multicolumn{6}{|c|}{ Área 2} \\
\hline 0 a 10 & 0,01 & 0,41 & 0,12 & 0,07 & 55,52 \\
\hline 10 a 20 & 0,05 & 1,60 & 0,97 & 0,23 & 23,85 \\
\hline 20 a 30 & 0,08 & 2,89 & 1,62 & 0,40 & 24,46 \\
\hline
\end{tabular}



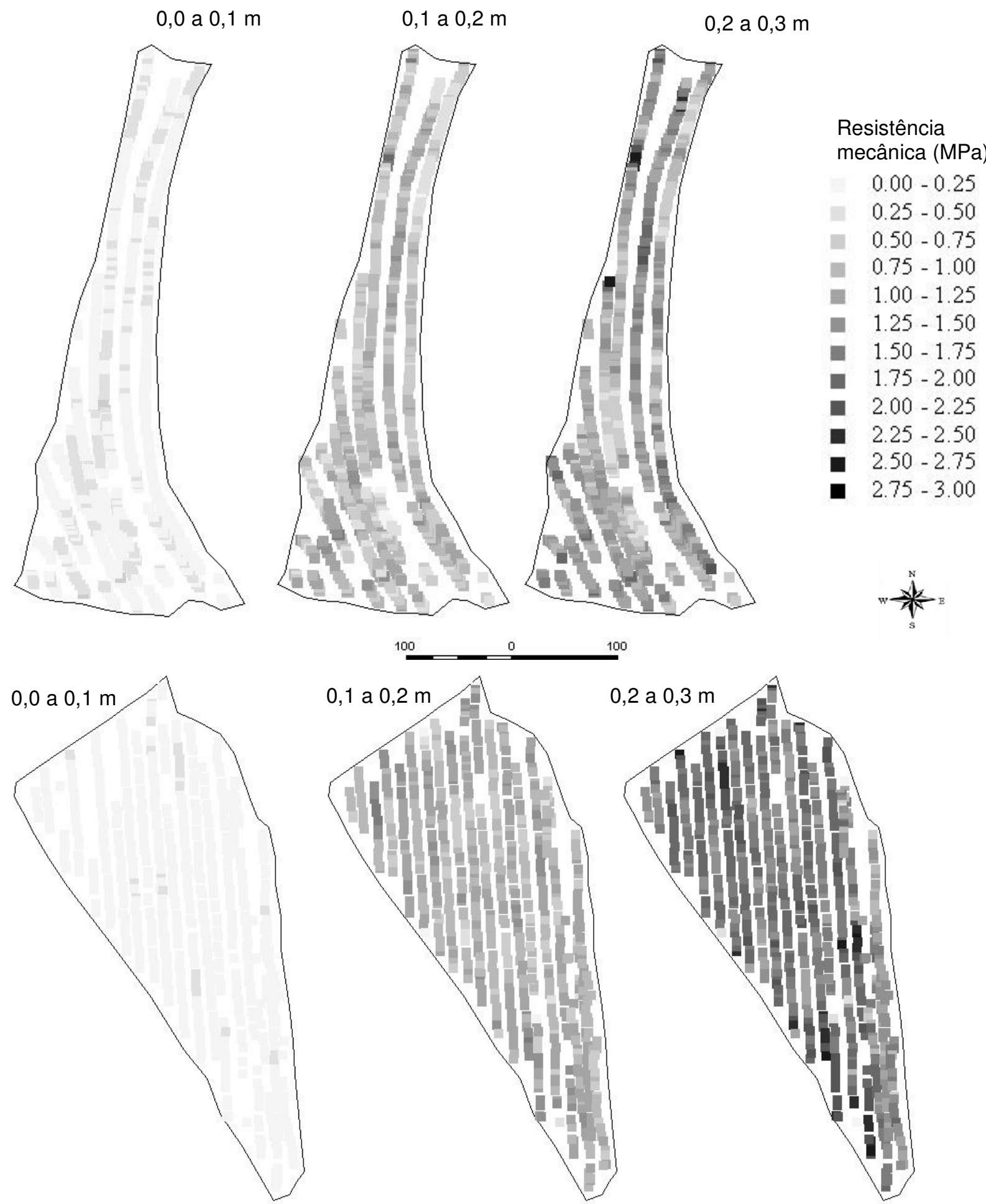

FIGURA 2. Mapas da resistência mecânica para cada camada de solo obtida com as respectivas hastes para as duas áreas experimentais. 


\section{CONCLUSÕES}

O sistema de múltiplas hastes instrumentadas com extensômetros de resistência elétrica permitiu a medida simultânea da resistência mecânica do solo em três profundidades, ao deslocar-se no campo.

A configuração mostrou ser mecanicamente simples, mesmo com três hastes independentes de mensuração de resistência mecânica do solo. Dados de testes em campo demonstraram que o sistema pode produzir mapas que indicam a variabilidade espacial da resistência mecânica das áreas amostradas, em três camadas, com resolução, densidade de amostragem e capacidade operacional mais elevada se comparada com levantamentos feitos com penetrômetros de cone.

\section{REFERÊNCIAS}

ADAMCHUK, V.I.; MORGAN, M.T.; SUMALI, H. Application of a strain gauge array to estimate soil impedance on-the-go. Transactions of the ASAE, St Joseph, v.44, n.6, p.1377-83, 2001.

ALIHAMSYAH, T.; HUMPHRIES, E.G.; BOWERS JR, C.G. A technique for horizontal measurement of soil mechanical impedance. Transactions of the ASAE, St Joseph, v.33, n.1, p.73-7, 1990.

ANDRADE, P.; ROSA, U.; UPADHYAYA, S.; JENKINS, B.; AGUERA, J.; JOSIAH, M. Soil profile force measurements using an instrumented tine. St. Joseph: ASAE, 2001. 10 p. (ASAE Paper No. 011060)

ASAE Standards S313.3. Soil cone penetrometer. St. Joseph, 2000a. 2 p.

EP542. Procedures for using and reporting data obtained with the soil cone penetrometer. St. Joseph, 2000b. 3 p.

BERTOL, I.; BEUTLER, J.F.; LEITE, D.; BATISTELA, O. Propriedades físicas de um cambissolo húmico afetadas pelo tipo de manejo do solo. Scientia Agrícola, Piracicaba, v.58, n.3, p.555-60, jul./set. 2001.

EHRHARDT, J.P.; GRISSO, R.D.; KOCHER, M.F.; JASA, P.J.; SCHINSTOCK, D.J.L. Using the Veris electrical conductivity cart as a draft predictor. St. Joseph: ASAE, 2001. 15 p. (ASAE Paper No. 011012)

FONSECA, M.M.; RAMOS, G.; PERALTA, U. Efecto de la compactación del suelo en tres cepas de caña (quedadad, frio y retoño), con tres modelos de cosechadoras (KTP-1, KTP-2, y TOFT-6000) y equipos de transporte. Atac, La Habana, v.1, n.1, p.7-17, 1988.

GILL, R.W. Influence of compaction hardening of soil on penetration resistance. Transactions of the $A S A E$, St. Joseph, v.11, n.6, p.741-5, 1968.

GORUCU, S.; KHALILIAN, A.; HAN, Y.J.; DODD, R.B.; WOLAK, F.J.; KESKIN, M. Variable depth tillage based on geo-referenced soil compaction data in coastal plain region of South Carolina. St. Joseph: ASAE, 2001. 11 p. (ASAE Paper No. 01-1016)

GLANCEY, J.L.; UPADHYAYA, S.K.; CHANCELLOR, W.J.; RUMSEY, J.W. An instrumented chisel for the study of soil tillage dynamics. Soil and Tillage Research, Amsterdam, v.14, n.1, p.1-24, 1989.

GLANCEY, J.L.; UPADHYAYA, S.K.; CHANCELLOR, W.J.; RUMSEY, J.W. Prediction of agricultural implement draft using an instrumented analog tillage tool. Soil and Tillage Research, Amsterdam, v.37, n.1, p.47-65, 1996. 
KLEIN, V.A. Propriedades físico-hídrico-mecânicas de um latossolo roxo, sob diferentes sistemas de uso e manejo. 1998. 150 f. Tese (Doutorado em Física do Ambiente Agrícola) - Escola Superior de Agricultura "Luiz de Queiroz", Universidade de São Paulo, Piracicaba, 1998.

LANÇAS, K.P.; NETO, P.C.; NAGAOKA, A.K.; GERRA, S.P.S. Índice de cone e mapas de isocompactação do solo agrícola, obtidos com a utilização de um pentrômetro hidráulico-eletrônico e um sistema de posicionamento global diferencial (DGPS). In: BALASTREIRE, L.A. O estado-da-arte da agricultura de precisão no Brasil. Piracicaba: L.A. Balastreire, 2000. p.113-23.

MAGALHÃES, R.P.; MOLIN, J.P.; FAULIN, G. DI C. Estudo da espacialidade do índice de cone em função do local amostrado em uma lavoura de café. In: CONGRESSO BRASILEIRO DE AGRICULTURA DE PRECISÃO, 2004, Piracicaba. Anais... Piracicaba: ESALQ/USP, 2004. p.1-7.

MANOR, G.; CLARK, R.L. Development of an instrumented subsoiler to map soil hard-pans and real-time control of subsoiler depth. St. Joseph: ASAE, 2001. 17 p. (ASAE Paper No. 011022)

MOLIN, J.P.; SILVA JUNIOR, R.L.D.A. Variabilidade espacial do índice de cone correlacionada com textura e produtividade. Engenharia Rural, Piracicaba, v.14, n.1, p.49-57, 2004

MORGAN, M.; ESS, D. The precision-farming guide for agriculturists. Moline: John Deere Publishing, 1997. 117 p.

OWEN, G.T.; DRUMMOND, H.; COBB, L.; GODWIN, R.J. An instrumented system for deep tillage research. Transactions of the ASAE, St. Joseph, v.30, n.6, p.1578-82, 1987.

RAPER, R.L.; WASHINGTON, B.H.; JARRELL, J.D. A tractor-mounted multiple-probe soil cone penetrometer. Applied Engineering in Agriculture, St. Joseph, v.15, n.4, p.287-90, 1999.

ROONEY, D.J.; DUDKA, M., CHEYNE, M.; SAMUELSON, J.R. The soil information system. St. Joseph: ASAE, 2002. 11 p. (ASAE Paper No. 021092)

SANTOS, J.C.F. Comportamento de propriedades físicas e químicas de dois latossolos roxos sob diferentes sistemas de rotação de culturas em plantio direto.1993. $101 \mathrm{f}$. Tese (Doutorado em Solos e Nutrição de Plantas) - Escola Superior de Agricultura de Lavras, Lavras,1993.

SIEFKEN, R.J.; ADAMCHUK, V.I.; EISENHAUER, D.E.; BASHFORD, L.L. Mapping soil mechanical resistance with a multiple blade system. Applied Engineering in Agriculture, St. Joseph, v.21, n.1, p.15-23, 2005.

SILVA JUNIOR, R.L. da. Variabilidade espacial do indice de cone correlacionada com mapas de produtividade. 2001. 132 f. Dissertação (Mestrado em Máquinas Agrícolas) - Escola Superior de Agricultura "Luiz de Queiroz", Universidade de São Paulo, Piracicaba, 2001.

SIRJACOBS, D.; HANQUET, B.; LEBEAU, B.; DESTAIN, M.F. On-line soil mechanical resistance mapping and correlation with soil physical properties for precision agriculture. Soil and Tillage Research, Amsterdam, v.64, p.231-42, 2002. 